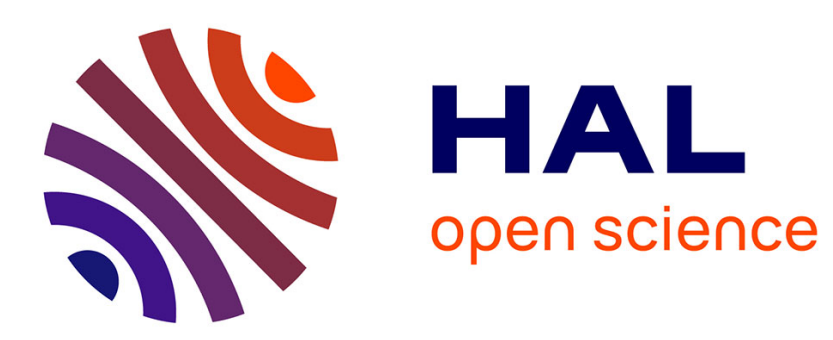

\title{
La mise à disposition des salariés sous-traitants chez les donneurs d'ordres: une source de malaise professionnel?
}

Christophe Everaere

\section{To cite this version:}

Christophe Everaere. La mise à disposition des salariés sous-traitants chez les donneurs d'ordres: une source de malaise professionnel?. Recherches en sciences de gestion, 2014, numéro 103, pp.1-23. halshs-01073477

\section{HAL Id: halshs-01073477 https://shs.hal.science/halshs-01073477}

Submitted on 9 Oct 2014

HAL is a multi-disciplinary open access archive for the deposit and dissemination of scientific research documents, whether they are published or not. The documents may come from teaching and research institutions in France or abroad, or from public or private research centers.
L'archive ouverte pluridisciplinaire HAL, est destinée au dépôt et à la diffusion de documents scientifiques de niveau recherche, publiés ou non, émanant des établissements d'enseignement et de recherche français ou étrangers, des laboratoires publics ou privés. 


\title{
La mise à disposition des salariés sous-traitants chez les donneurs d'ordres : une source de malaise professionnel ? *
}

\author{
* Revue Recherches en Sciences de Gestion, \\ numéro 103/2014, novembre 2014 \\ Christophe EVERAERE \\ Professeur des Universités \\ IAE - Université Jean Moulin Lyon 3 \\ Centre de recherche Magellan \\ 6 cours Albert Thomas \\ 69355 Lyon Cedex 08
}

Tel. 0637780412

e-mail : christophe.everaere@univ-lyon3.fr

\section{Résumé :}

Le développement des pratiques de sous-traitance donne à voir des situations particulières dans lesquelles les salariés d'entreprises prestataires de services sont mis à disposition des entreprises clientes donneuses d'ordres. Contrairement au modèle de l'entreprise flexible de J. Altkinson, nous avons découvert, grâce aux données statistiques de l'enquête COI 2006, que les salariés concernées par ces mises à disposition sont durablement hébergés chez les clients et occupent pour une partie importante d'entre eux des postes à responsabilité. Selon les déclarations des intéressés, leur situation hybride, à fois sur des postes à forts enjeux, mais sans faire partie des effectifs de l'entreprise où ils sont affectés, soulèvent deux problèmes majeurs que sont le sentiment d'isolement et une incertitude quant à leur avenir professionnel. Il en découle une forme de malaise professionnel qui détériore leur implication dans le travail.

Mots-clés : externalisation, mise à disposition, insiders, outsiders.

Summary :

To gain flexibility, companies resort to atypical forms of employment such as the fixed-term contracts, the interim, or the part-time work. Other forms of atypical employment also exist over which we have less information especially those that 
induce an employee to work in the customer's premises within a subcontracting relationship. What is called seconded staff.

The statistics out of the survey "Organizational Change and Informatics" have enabled both to assess the extent of this phenomenon, identify the characteristics of this particular population, and also to question the adequacy of this particular form of employment with the required skills. It is clear that a significant portion of the seconded staff (employees from the subcontractors who work in the customers' premises) performs work that is as qualified and important that the "inside employees", but with an outsider status. This is a hybrid situation that puts them in trouble and disturbs in particular their involvement in the work.

Key-words:, outsourcing, seconded staff, insiders, outsiders.

\section{Remerciements :}

Une version préliminaire de ce texte a été présentée au XXIIe congrès de l'AGRH à Marrakech (26-28 octobre 2011) avec Mireille LapoireChasset sous le titre : «Le salarié mis à disposition: un insider externalisé ». Nous avons exploité ensemble une partie des données statistiques présentées dans le papier. Mais je suis seul responsable de la nouvelle analyse orientée sur la question du malaise professionnel et des éventuelles erreurs qu'elle comporte. Qu'elle soit ici remerciée de sa collaboration.

Cette proposition d'article a pu être rédigée grâce à l'expertise informatique et statistique de Pierre-Olivier Chasset qui a assuré le traitement des données de l'enquête Changement Organisationnel et Informatique (COI), à la base de ce travail. Qu'il en soit ici remercié.

Nous remercions également le Centre d'Etudes de 1'Emploi et Sylvie Hamont-Cholet, en particulier, pour nous avoir permis d'accéder aux données de l'enquête COI 2006 et nous avoir conseillé dans leur analyse et leur traitement statistique ; ainsi qu'ARAVIS qui a apporté également son soutien à la réalisation de cette étude.

\section{Introduction}

Il est question d'envisager ici la situation particulière des salariés qui travaillent, non pas chez leur employeur avec qui une relation d'emploi (et donc un contrat de travail) a été établi, mais chez le ou les clients de ce dernier, sur des durées parfois longues (plusieurs années) et à temps plein. Un peu comme s'il y avait un double employeur : l'employeur "de droit" (celui qui emploie, rémunère, mais que 
le salarié voit rarement) et l'employeur "de fait", celui chez qui le salarié travaille effectivement et de façon potentiellement régulière, mais sans en être salarié(e).

Cette mise à disposition rentre dans le cadre d'une relation de sous-traitance particulière selon laquelle la prestation de service est réalisée dans les locaux de l'entreprise cliente. C'est le cas notamment des informaticiens dits 'en régie' employés par des SSII (Sociétés de Services en Ingénierie Informatique) qui s'occupent des systèmes d'information implantés dans les locaux de l'entreprise cliente, ou des femmes de ménage qui assurent le nettoyage des établissements des entreprises clientes de leur employeur.

Se posent ici plusieurs questions importantes pour ces salariés atypiques : à quelle entreprise ces salariés mis à disposition s'identifient-ils ? Ont-ils le sentiment d'être complètement intégrés dans les collectifs de travail de l'entreprise où ils interviennent ? Sont-ils des collègues comme les autres, membres à part entière des équipes où ils sont mis à disposition ? S'impliquent-ils autant que les salariés qui travaillent à demeure chez leur employeur ? Eprouvent-ils un sentiment de précarité du fait de leur situation ambiguë ? Comment ces salariés se projettent-ils dans l'avenir sachant que leur mise à disposition est soumise à une relation commerciale (la prestation de service qui justifie leur présence dans les locaux de l'entreprise cliente) ; mais que cette relation commerciale comporte une échéance dont le renouvellement n'est pas garanti ? En effet, même s'ils sont en CDI avec l'entreprise qui les a recrutés, qu'advient-il d'eux quand les mises à disposition s'achèvent : une nouvelle mise à disposition ?; mais où ?; pour faire quoi ?; avec qui ? comment ? ; et que se passe-t-il si les périodes de non mise à disposition se prolongent, etc. ? Autant d'incertitudes potentiellement perturbantes.

L'exploitation des résultats de l'enquête Changement Organisationnel et Informatique (COI) 2006 réalisée auprès des salariés des entreprises privées de plus de 20 personnes ${ }^{1}$ a permis d'évaluer l'ampleur et le ressenti de cette forme de travail atypique que constitue la mise à disposition (MAD) chez ceux qui la vivent.

Ainsi 5,4\% des personnes interrogées dans le cadre de cette enquête (soit 769 personnes sur un échantillon total de 14247 salariés interrogés) sont employées par des entreprises privées pour être mises à disposition d'autres sociétés, en dehors du cadre particulier du travail intérimaire.

\footnotetext{
${ }^{1}$ L'enquête COI a été menée conjointement par le Centre d'Etudes de l'Emploi (CEE), le Service des Etudes et des Statistiques Industrielles (SESSI), le Service Central des Enquêtes et Études Statistiques (SCESS), la Direction de l'Animation de la Recherche, des Etudes et des Statistiques (DARES) et l'Institut National de la Statistique et des Etudes Economiques (INSEE), auprès des entreprises privées de plus de 20 salariés et de leurs salariés. Cette enquête comporte ainsi deux volets : celui des entreprises et celui des salariés. Les résultats présentés et analysés ici sont extraits de ce deuxième volet (les salariés).
} 
La question exacte ayant permis d'isoler cette population était formulée en ces termes (question 4ter du questionnaire principal auprès des salariés) : «En excluant la situation particulière du contrat d'intérim, êtes-vous dans un des cas suivants : 1. Mis à disposition par votre employeur dans une autre entreprise (type travail en régie, au forfait)? 2. En mission permanente dans différentes entreprises (type mission d'expertise dans plusieurs entreprises, y compris services de nettoyage dans plusieurs entreprises) ?». Ne sont pas prises en compte ici les situations de mise à disposition au domicile des particuliers, inhérentes aux activités de services à la personne.

Les nombreuses questions qui leur ont été posées concernant leur profil, leur qualification, la nature de leur travail, leur niveau d'autonomie, le type de contrôle et de coopération, leur implication dans le travail, etc., ont livré des résultats surprenants.

En effet, alors que la théorie de la segmentation du marché du travail (insiders / outsiders), reprise dans le modèle de l'entreprise flexible, stipule que les soustraitants sont supposés être mobilisés pour de courtes périodes afin de répondre à une demande temporaire et/ou occuper des postes nécessitant une faible qualification largement disponible sur le marché du travail, $45 \%$ des salariés MAD sont placés durablement dans la même entreprise utilisatrice, sur des postes à forte responsabilité, nécessitant un niveau de qualification élevé, voire très élevé.

Selon les déclarations des intéressés, leur situation hybride, à fois sur des postes à forts enjeux, mais sans faire partie des effectifs de l'entreprise où ils sont affectés, soulèvent deux problèmes majeurs qui viennent remettre en cause les vertus de cette externalisation in situ (dans les locaux de l'entreprise cliente) et « haut de gamme » s'agissant des personnes qualifiées dont il est question parmi les MAD.

Ces deux problèmes majeurs concernent tout d'abord le sentiment d'isolement ou d'exclusion des collectifs de travail exprimés par les MAD : isolement qui contredit l'exigence de coopération nécessaire pour tout collaborateur en général dans une entreprise, mais encore plus pour des cadres et des managers qui représentent une partie non négligeable de la population des MAD. Le deuxième problème majeur repéré au travers de l'enquête est celui d'un sentiment de précarité et d'une implication mitigée dans le travail exprimée par les MAD. Ces deux reproches, étayés par des verbatim de personnes concernées par la mise à disposition, relèvent d'une forme de malaise professionnel qui nous semble peu compatible avec l'exercice de responsabilités importantes dans l'entreprise où ces personnes sont mises à disposition. Ceci nous amène à débattre des limites de la flexibilité externe induite par cette externalisation hybride qui conduit à dissocier la relation d'emploi et les relations de travail.

Après avoir défini cette forme atypique d'emploi et énoncé quels usages l'entreprise flexible est supposée en faire (1.), nous verrons à quel point les travailleurs externalisés de cette manière correspondent peu au profil attendu (2.), et dans quelle mesure leur situation singulière pose à la fois des problèmes 
d'intégration dans des collectifs de travail, d'implication pour eux et donc de gestion pour les entreprises utilisatrices (3.).

\section{La mise à disposition : une forme particulière d'externalisation}

Le salarié mis à disposition est employé par une entreprise, en CDD ou en CDI, à temps complet ou à temps partiel, pour être délégué au service d'une ou plusieurs autres entreprises, dans le cadre d'un contrat de sous-traitance dont la durée peut être courte ou longue : «Il y a mise à disposition du salarié lorsque celui-ci, avec ou sans son consentement, est appelé par son employeur à accomplir sa prestation de travail auprès d'une autre entreprise à l'exploitation de laquelle il concourt » (Lyon-Caen et De Maillard, 1981, p. 320).

Selon les chiffres de l'enquête COI 2006, 78 \% des salariés MAD occupent un emploi en CDI. La moitié est mise à disposition d'une seule et même entreprise, dans le cadre d'un travail en régie par exemple ${ }^{2}$. L'autre moitié est constituée de salariés en mission permanente dans différentes entreprises, pour mener, par exemple, des missions d'expertise ou assurer un service de nettoyage. Dans les deux cas, le salarié MAD est ainsi amené à travailler dans les locaux de l'entreprise cliente, aux côtés de son personnel, quelquefois même au sein de ses équipes, mais sans appartenir à ses effectifs.

Ce phénomène de la mise à disposition est donc directement lié aux pratiques d'externalisation (ou outsourcing) qui consistent pour des entreprises à se défaire d'activités qu'elles ne considèrent pas ou plus faire partie de leur cœur de métier ${ }^{3}$. Lesquelles activités sont alors sous-traitées si elles sont plutôt ponctuelles et facilement réversibles, ou alors externalisées si les activités qui sortent ainsi du périmètre de l'entreprise s'inscrivent dans des relations plus durables avec des contrats de prestations qui peuvent s'étendre sur plusieurs années. Avec la mise à disposition, nous sommes dans un cas de figure original d'externalisation selon lequel l'entreprise « cliente » héberge en son sein, sur des durées parfois longues (plusieurs années), le personnel d'une entreprise extérieure à laquelle elle achète

\footnotetext{
${ }^{2}$ Le travail en régie consiste pour une entreprise (souvent informatique) à fournir non pas la garantie d'un résultat comme dans le travail au forfait, mais une garantie de moyens, en l'occurrence humains, que l'entreprise affecte en général chez le client, sur une période généralement assez longue, avec facturation à la durée de séjour du salarié chez le client. Le salarié de l'entreprise (informatique) est donc mis à disposition du client chez qui il travaille en suivant ses rythmes pour résoudre les problèmes techniques ou de mise en œuvre des solutions informatiques (Everaere et al., 2003).

3 Les publications sur le sujet de la sous-traitance (plutôt ponctuelle menée avec des PME) ou de l'externalisation (plutôt menée avec des grandes entreprises sur des durées plus longues) sont très nombreuses, depuis les travaux fondateurs de PY. Barreyre (1992), ceux de M. Capet (1989) en France. Il n'est pas possible d'en faire ici une recension exhaustive. Retenons l'idée générale que la sous-traitance ou l'externalisation s'inscrit dans une logique qui combine plusieurs objectifs : la recherche de flexibilité, la recherche de productivité (réduction des coûts) et de qualité par la spécialisation de chacun, l'accès à des expertises non disponibles en interne. Même si les résultats concrets ne sont pas toujours à la hauteur des espérances comme le montrent les enquêtes TNS Sofrès du baromètre de l'externalisation menées par Ernst et Young.
} 
des prestations (de conseil, d'informatique, de maintenance, de gardiennage, de nettoyage, etc. ${ }^{4}$ ) ; alors que dans une sous-traitance plus classique, de type industriel, l'activité confiée par le donneur d'ordre est réalisée dans les locaux du sous-traitant, sous la surveillance et le contrôle de ce dernier.

De ce point de vue, la mise à disposition s'apparente au travail intérimaire : les salariés MAD, comme les intérimaires, constituent pour l'entreprise utilisatrice une main-d'œuvre externe mais présente sur place : employés par des prestataires de service ou des entreprises de travail temporaire, ces collaborateurs atypiques travaillent dans les locaux de l'entreprise utilisatrice pour travailler avec ses propres salariés.

Cependant, la mise à disposition (dans le cadre d'une relation de sous-traitance ou d'externalisation) diverge de l'intérim d'un point de vue juridique. Officiellement, la location de main d'œuvre à but lucratif ne peut être réalisée que par des entreprises de travail temporaire (article L 125 - 3 de la loi du 3 janvier 1972), sinon il y a délit de marchandage ou prêt illicite de main d'œuvre.

En théorie, la sous-traitance et la prestation de service, moyennant un détachement de main d'œuvre (c'est le cas particulier qui nous intéresse ici des salariés MAD), n'ont pas pour effet de soumettre cette main d'œuvre à l'autorité directe du donneur d'ordre. «En d'autres termes, dans le contrat de prestation de service ou de sous-traitance, le donneur d'ordres est supposé ignorer sur le plan juridique le salarié qui exécute la prestation commandée : le donneur d'ordres demande la réalisation d'un travail dont il assure la réception après son exécution, sans se préoccuper de la façon dont la prestation est effectuée, du nombre de salariés affectés, de leur qualification professionnelle, et des moyens mis en œuvre pour exécuter le travail » (Maillard de J. et al. 1979, p. 327). Mais il est évident que cette disposition est difficilement applicable dans le cas de salariés mis à disposition chez l'entreprise cliente qui achète une prestation et qui va vraisemblablement s'adresser directement à la personne mise à sa disposition pour lui donner du travail, lui donner des consignes, éventuellement lui procurer des outils ou des équipements, et donc exercer sur cette personne une forme d'autorité.

Dans le texte déjà cité (Maillard de J. et al., 1979), plusieurs juristes en France s'étonnaient il y a déjà plus de trente ans, des pratiques de sous-traitance et de leurs impacts sur l'éclatement de la collectivité de travail au sein des entreprises utilisatrices. Ils parlaient même de «mosaïque de personnels » réunis en un même lieu sous la dépendance du chef de l'entreprise cliente, tout en relevant d'entreprises juridiquement et économiquement distinctes.

Sans faire juridiquement partie des effectifs de l'entreprise cliente, le salarié mis à disposition constitue pour cette dernière une ressource humaine (externe mais

\footnotetext{
${ }^{4}$ Selon les données de l'enquête COI, les employeurs des salariés mis à disposition relèvent effectivement de secteurs divers : principalement les services d'assistance et de conseil, les services opérationnels, la construction et les transports.
} 
présente sur place) qui doit se plier aux règlements, aux horaires, aux rythmes et aux conditions de travail de l'entreprise cliente.

En apparence, rien ne distingue les salariés internes titulaires et ces collaborateurs externes mis à disposition qui travaillent ensemble, ou tout au moins à proximité, au même endroit. Cependant, au vu des témoignages que nous restituerons ciaprès, nous verrons que cette relation particulière d'emploi que constitue la mise à disposition n'est pas neutre dans les relations au travail entre «collègues» et qu'elle peut même induire une certaine mise à distance, qui nous semble préjudiciable à la nécessaire coopération et efficacité dans le travail.

\section{Les salariés mis à disposition : des outsiders atypiques ?}

Dans les représentations les plus courantes de la flexibilité, la sous-traitance à laquelle correspond la pratique de salariés externes mis à disposition dans l'entreprise cliente est clairement située au même niveau que l'intérim, c'est-àdire dans l'ellipse la plus éloignée du noyau central des salariés titulaires (les insiders), comme le montre la modélisation de la firme flexible proposée par J. Atkinson (1984, p. 29) dans la figure 1.

Cette terminologie insider-outsider est empruntée à la théorie de la segmentation (Doeringer et Piore, 1971) qui oppose un marché interne (il s'agit globalement des salariés stables durablement embauchés par l'entreprise car détenteurs de compétences critiques ou spécifiques à l'entreprise), et un marché externe (les outsiders) correspondant à des salariés sans perspective de carrière, le plus souvent faiblement qualifiés, et dont les séjours en entreprises sont brefs et irréguliers (Gazier, 2003).

Figure $1 \quad-\quad$ L' « entreprise flexible » d'après J. Atkinson

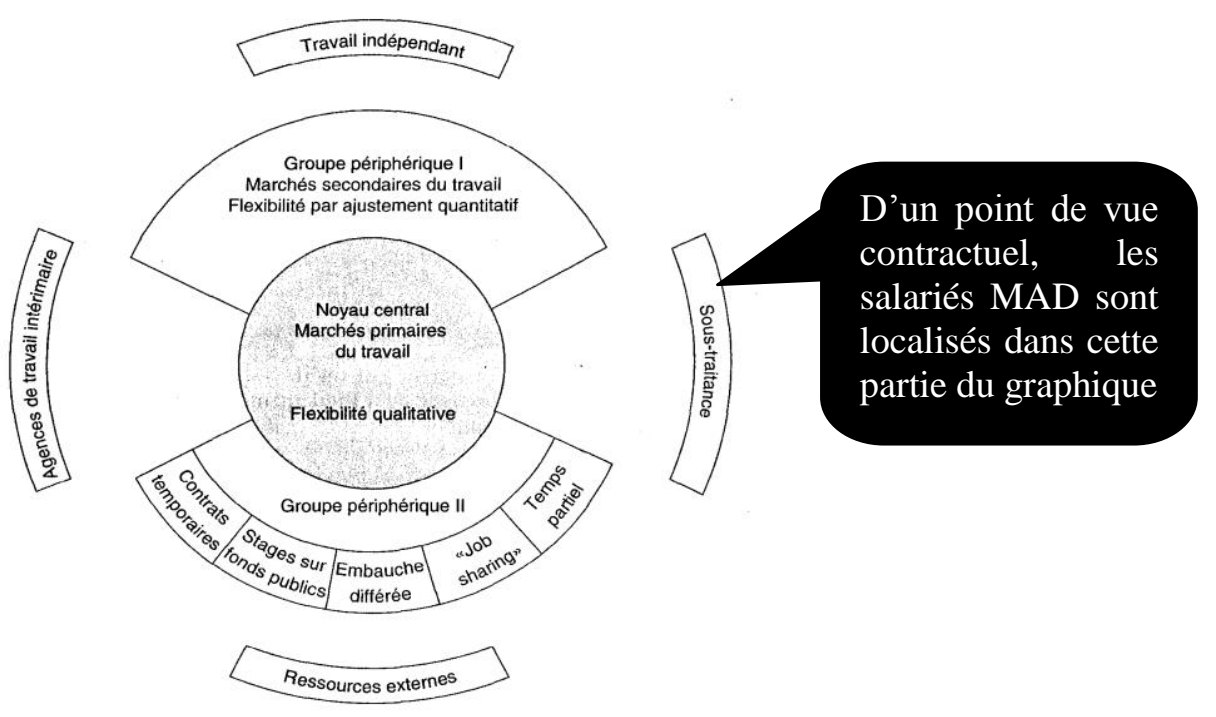


Dans ces ressources humaines externes, conformément à la théorie des coûts de transaction (Williamson, 1975), on ne devrait trouver que des ressources ayant une double caractéristique.

- Elles devraient être faiblement spécifiques, c'est-à-dire facilement disponibles sur le marché, ou capables d'occuper des postes rapidement dans l'entreprise, en raison du faible niveau de qualification requis par ces postes.

- L'entreprise ne devrait pas avoir besoin souvent ou de façon durable de ces ressources (externes). Dans les termes de la théorie des coûts de transaction, la fréquence de transaction devrait être faible.

Ce sont les deux raisons principales pour lesquelles, s'agissant de ces ressources humaines externes les plus périphériques, l'entreprise recourt - en principe - à une régulation marchande, autrement dit à un lien commercial, au lieu d'établir un contrat salarial, comme c'est le cas pour les autres ressources humaines qui sont internalisées (embauchées en CDI directement par l'entreprise utilisatrice).

C'est une distinction très importante par rapport aux autres catégories de salariés. Un intérimaire, par exemple, ne fait pas partie des effectifs de l'entreprise cliente qui recourt aux services d'une entreprise de travail temporaire. Il en est de même pour les sous-traitants dont les salariés sont implantés chez l'entreprise cliente mais sans faire partie de ses effectifs (ce qui est le cas de la population à laquelle nous nous intéressons ici des salariés MAD), ou les travailleurs indépendants, tels que des avocats ou des consultants (en profession libérale) qui interviennent ponctuellement en entreprise ou des chauffeurs routiers (indépendants) à qui telle entreprise confie des prestations de transport en fonction de ses propres besoins, à nouveau sur une base plutôt ponctuelle ou occasionnelle.

Mobilisant la théorie des ressources (core competences) de Hamel et Prahalad qu'il articule avec le modèle de la firme flexible d'Atkinson, L. Cadin (1997) confirme que la sous-traitance est associée à un type de compétence qualifiée de secondaire ou accessoire (Maillard et al. 1979), par opposition au type de compétence qualifiée de fondamentale qui justifie le recours au contrat à durée indéterminée propre aux insiders.

Cependant, en comparant les caractéristiques des travailleurs, l'enquête COI 2006 montre que si la main-d'œuvre intérimaire correspond peu ou prou au modèle d'Atkinson (des personnes peu qualifiées mobilisées sur des missions courtes ${ }^{5}$ ), en revanche, les salariés mis à disposition s'en distinguent très clairement. Non seulement, près de la moitié est déléguée durablement auprès d'une seule et même entreprise, mais leur profil et leur position dans l'organisation des entreprises utilisatrices sont très différentes de celle des intérimaires et ressemblent au contraire à ceux des salariés en CDI, employés à temps complet

\footnotetext{
${ }^{5}$ La durée moyenne d'une mission d'intérim est d'un peu moins de deux semaines et un quart des missions ne dépasse pas la journée (Finot, 2012).
} 
pour travailler à demeure. Autrement dit, bien que les entreprises aient externalisé les emplois des MAD, elles leur confient les mêmes responsabilités qu'à leurs salariés permanents à temps complet, qui constituent, dans le modèle, le « cœur de l'entreprise » et, qu'à ce titre, elles sont supposées intégrer et conserver.

\section{Encadré 1 - Méthodologie de comparaison des différentes formes d'emploi grâce aux données de l'enquête COI}

L'enquête C.O.I. (Changement Organisationnel et Informatisation) est un dispositif d'enquêtes couplées entreprises / salariés sur les changements organisationnels et l'informatisation. Le volet « entreprises » de l'enquête cherche à cerner la manière dont l'entreprise mobilise les outils de gestion de l'organisation du travail et des ressources humaines ainsi que les outils techniques couramment regroupés sous les termes de TIC (Technologies de l'Information et de la Communication). La collecte auprès des salariés (que nous exploitons ici) apporte une information complémentaire au volet « entreprises » sur les caractéristiques organisationnelles des postes de travail et sur les usages des TIC. Cette enquête permet d'évaluer les effets des changements dans l'organisation et l'outillage informatique des entreprises sur l'organisation du travail des salariés, leurs compétences, leur formation, les conditions de travail, les salaires.

Le volet « salariés " de l'enquête a été réalisé par téléphone ou, à défaut, en face à face auprès de deux salariés de l'entreprise pour les entreprises de moins de 500 salariés, et auprès de davantage de salariés pour les très grandes entreprises (13 au maximum), de façon à avoir en moyenne 3 salariés interrogés par entreprise. Les salariés interrogés ont fait l'objet d'un tirage aléatoire à partir des fichiers issus des DADS (déclaration annuelle des données sociales) des entreprises.

En recueillant un riche panel de données sur les conditions de travail, les responsabilités, l'entraide et l'autonomie dans le travail... auprès de 14247 salariés employés dans des entreprises privées de plus de 20 personnes, l'enquête COI 2006 a permis d'isoler un effectif suffisamment large de salariés concernés par la mise à disposition (769 personnes, soit 5,4\% de l'ensemble de l'échantillon interrogé). En procédant par tri à plat, nous avons dans un premier temps identifié les caractéristiques démographiques des personnes concernées par la MAD (genre, âge, niveau d'études, CSP, type d'emploi). Dans un second temps, pour les questions relatives à l'organisation du travail (coopération, responsabilité, autonomie, sentiment de fragilité professionnelle, degré d'implication dans le travail, etc.) nous avons comparé les réponses fournies par les salariés en MAD avec d'une part les salariés employés à temps plein en CDI à demeure qui occupent les emplois typiques et qui constituent le noyau central de l'entreprise flexible, et pour les comparer d'autre part aux autres salariés internes interrogés en emploi atypique, employés à temps partiel, à durée déterminée, en stage rémunéré ou en contrat aidé qui sont supposés constituer la première périphérie entre le «noyau central » et les travailleurs externes.

Par contre, concernant la population des intérimaires, le nombre d'intérimaires interrogées dans l'enquête COI n'étant pas suffisant, nous avons travaillé avec les données démographiques exhaustives disponibles à partir des enquêtes Emploi de l'INSEE et des résultats de l'exploitation par la DARES des fichiers UNEDIC des déclarations mensuelles des agences d'intérim. 
S'ils ont des statuts similaires, intérimaires et salariés MAD n'ont donc pas le même profil. En effet, alors que la figure emblématique du travailleur intérimaire est celle d'un homme jeune (les deux tiers ont moins de 35 ans), le salarié MAD est un homme d'âge plus avancé, comparable aux salariés internes employés à temps complet et à durée indéterminée (les deux tiers ont plus de 35 ans). De même, tandis que l'intérimaire type n'est pas ou peu diplômé (38\% ont au maximum le BEPC, $38 \%$ ont suivi une filière de formation professionnelle courte et seulement $10 \%$ sont diplômés de l'enseignement supérieur), les MAD, comme les permanents à temps complet, sont plus nombreux à avoir suivi de longues études ( $35 \%$ ont un niveau BEP/CAP et $34 \%$ ont un diplôme post baccalauréat).

Les postes confiés aux uns et aux autres ne sont pas non plus les mêmes. Tandis que $80 \%$ des intérimaires exercent un métier d'ouvrier, les MAD ainsi que les salariés internes à temps complets ne sont que $30 \%$ dans ce cas. Les autres occupent à part sensiblement égale des postes d'employés (27\% pour les MAD et $23 \%$ pour les permanents), des professions intermédiaires (respectivement $22 \%$ et $24 \%$ ) et des postes de cadres $(21 \%$ pour les MAD et $23 \%$ pour les salariés internes à temps complet, alors que les cadres représentent moins de $2 \%$ de la population des intérimaires ${ }^{6} c f$. tableau 1$)$.

\footnotetext{
${ }^{6}$ Le pourcentage d'intérimaires ayant le statut de cadre est de $1,8 \%$ et ce taux n'a jamais dépassé les $2 \%$ depuis au moins 10 ans (Domens, 2010).
} 
Tableau 1: Répartition en pourcentage des salariés par type d'emploi occupé, par âge, sexe, qualification et niveau d'études

\begin{tabular}{|c|c|c|c|c|c|}
\hline & \multirow{2}{*}{$\begin{array}{c}\text { Salariés } \\
\text { intérimaire } \\
\mathrm{s}\end{array}$} & \multicolumn{4}{|c|}{ Salariés des entreprises de plus de 20 personnes } \\
\hline & & MAD & $\begin{array}{c}\text { Salariés } \\
\text { internes en } \\
\text { CDI à temps } \\
\text { complet }{ }^{*} \\
\end{array}$ & $\begin{array}{c}\text { Autres } \\
\text { salariés } \\
\text { internes } * *\end{array}$ & $\begin{array}{c}\begin{array}{c}\text { Total } \\
\text { des } \\
\text { salariés } \\
\text { internes }\end{array} \\
\end{array}$ \\
\hline \multicolumn{6}{|l|}{ Sexe } \\
\hline Hommes & 72 & 73 & 70 & 23 & 66 \\
\hline Femmes & 28 & 27 & 30 & 77 & 34 \\
\hline \multicolumn{6}{|l|}{ Age } \\
\hline Moins de 20 ans & 4 & 0 & 0 & 3 & 1 \\
\hline De 20 à 34 ans & 62 & 33 & 27 & 36 & 28 \\
\hline De 35 à 49 ans & 27 & 52 & 50 & 42 & 49 \\
\hline Plus de 50 ans & 7 & 15 & 23 & 20 & 22 \\
\hline \multicolumn{6}{|l|}{ Niveau d'études (dernier diplôme obtenu) } \\
\hline Primaire & 31 & 8 & 5 & 6 & 5 \\
\hline Secondaire 1er cycle & 7 & 8 & 6 & 9 & 6 \\
\hline Technique cycle court : préparation CAP, BEP & 38 & 35 & 36 & 26 & 35 \\
\hline Secondaire générale (seconde à la terminale) & & 8 & 9 & 11 & 9 \\
\hline Préparation d’un bac professionnel & 14 & 7 & 9 & 10 & 9 \\
\hline Supérieur 1er cycle (y compris BTS, IUT,...) & 8 & 15 & 17 & 19 & 17 \\
\hline Supérieur 2nd cycle & 2 & 8 & 6 & 9 & 6 \\
\hline Supérieur 3ème cycle & & 5 & 6 & 7 & 6 \\
\hline Grande école, d'ingénieur, de commerce & & 6 & 7 & 4 & 6 \\
\hline \multicolumn{6}{|l|}{ CSP } \\
\hline Manœuvre ou ouvrier spécialisé & 39 & 11 & 7 & 11 & 8 \\
\hline Ouvrier qualifié ou hautement qualifié & 40 & 19 & 23 & 8 & 21 \\
\hline Employé & 12 & 27 & 23 & 58 & 26 \\
\hline Professions intermédiaires & 7 & 22 & 24 & 14 & 23 \\
\hline Agent de maîtrise, maîtrise admin. ou commerciale & & 8 & 13 & 6 & 12 \\
\hline Technicien & & 14 & 11 & 7 & 11 \\
\hline Cadre & 2 & 21 & 23 & 10 & 22 \\
\hline Ingénieur ou cadre (hors cadres dirigeants) & & 20 & 22 & 10 & 21 \\
\hline Directeur général, adjoint direct & & 1 & 1 & 0 & 1 \\
\hline Total & 100 & 100 & 100 & 100 & 100 \\
\hline \multicolumn{6}{|c|}{$\begin{array}{l}\text { * Salariés internes donc à demeure, en CDI à temps complet, constituant le " noyau dur » dans le modèle } \\
\text { d'Atkinson. }\end{array}$} \\
\hline \multicolumn{6}{|c|}{$\begin{array}{l}\text { Sources: Répartition des intérimaires selon leur sexe, leur âge et leur CSP d'après les données UNEDIC 2006, } \\
\text { traitées par la DARES. Répartition des intérimaires selon leur niveau de diplôme d'après l'enquête } \\
\text { emploi en continu de l'INSEE. } \\
\text { Autres répartitions d'après l'enquête COI } 2006 \text { à partir de laquelle nous avons mené nos } \\
\text { traitements. }\end{array}$} \\
\hline
\end{tabular}

Les entreprises confient aux MAD des postes à risques au moins aussi souvent qu'aux salariés internes à temps complet (insiders). En effet, les MAD sont légèrement plus nombreux que les salariés internes à temps complet à affirmer qu'une erreur dans leur travail pourrait entraîner des conséquences dangereuses pour leur sécurité ( $46 \%$ contre $40 \%$ ) et celles d'autres personnes (47\% contre $42 \%$ ), et entraîner d'autres conséquences négatives pour eux (54 \% contre $51 \%$ ), pour d'autres personnes (31\% contre $29 \%$ ) et surtout pour l'entreprise (76\% contre $68 \%$ ) ( $c f$ tableau 2).

Par ailleurs, les entreprises confient aux MAD au moins autant de responsabilité d'équipe qu'aux «permanents». Ils sont ainsi presque aussi nombreux que les 
salariés en CDI à temps complet à occuper des places de chef d'équipe (30 et $32 \%$ ) et deux fois plus nombreux que les autres «permanents », en CDD ou à temps partiel $(12 \%)$. Les attributions des chefs MAD sont aussi très étendues : comparables à celles des chefs en CDI employés à temps plein, elles sont beaucoup plus conséquentes que celles des autres chefs en CDD, ou à temps partiel. Ainsi, ils sont tous sollicités de manière comparable pour expliquer le travail à réaliser à leurs subalternes. Mais les chefs MAD, tout comme les chefs permanents en CDI, sont deux fois plus nombreux que les autres chefs à temps partiel ou en CDD à répartir souvent le travail entre leurs subalternes (32 et $31 \%$ contre $15 \%$ pour les autres salariés internes), à régler souvent leurs problèmes et autres conflits $(32 \%$ contre $18 \%)$ et à évaluer leur travail (13 et $15 \%$ contre $4 \%$ ). Les chefs MAD et les chefs permanents en CDI sont aussi plus nombreux que les autres chefs à pouvoir influencer sur l'augmentation ou la promotion de ses subalternes (57 et $55 \%$ contre $32 \%$ ). Autrement dit, comme les chefs permanents en CDI et à temps complet, les salariés MAD exercent une autorité hiérarchique, alors même qu'ils ne sont pas salariés de l'entreprise où ils remplissent ce type de responsabilité importante ( $c f$ tableau 2).

Tableau 2 - Répartition en pourcentage des salariés des entreprises de plus de 20 personnes selon leur type d'emploi et selon les enjeux et les responsabilités managériales liés à leur poste

\begin{tabular}{|c|c|c|c|c|}
\hline & MAD & $\begin{array}{l}\text { Salariés internes } \\
\text { en CDI à temps } \\
\text { complet }\end{array}$ & $\begin{array}{l}\text { Autres } \\
\text { salariés } \\
\text { Internes }\end{array}$ & $\begin{array}{c}\text { Moyenne } \\
\text { pondérée des } \\
\text { salariés internes }\end{array}$ \\
\hline $\begin{array}{l}\text { Enjeux du poste occupé } \\
\text { Salariés dont une erreur dans le travail peut entraîner : } \\
\text { - des conséquences dangereuses pour votre sécurité } \\
\text { - des conséquences dangereuses pour la sécurité d'autres } \\
\text { - des conséquences négatives autres pour vous } \\
\text { - des conséquences négatives autres pour d'autres pers. } \\
\text { - des conséquences négatives autres pour l'entreprise } \\
\text { (pertes financières, mauvaise image, perte de clients...) }\end{array}$ & $\begin{array}{l}46 \\
47 \\
54 \\
31 \\
76\end{array}$ & $\begin{array}{l}40 \\
42 \\
51 \\
29 \\
68\end{array}$ & $\begin{array}{l}27 \\
27 \\
47 \\
18 \\
57\end{array}$ & $\begin{array}{l}39 \\
41 \\
50 \\
28 \\
68\end{array}$ \\
\hline $\begin{array}{l}\text { Management d'équipe } \\
\text { Salariés chefs d'équipes }\end{array}$ & 30 & 32 & 12 & 30 \\
\hline $\begin{array}{l}\text { Responsabilités managériales des chefs d'équipes } \\
\text { Répartition du travail entre collègues } \\
\text { Souvent } \\
\text { Parfois } \\
\text { Jamais }\end{array}$ & $\begin{array}{l}32 \\
30 \\
38\end{array}$ & $\begin{array}{l}31 \\
27 \\
43\end{array}$ & $\begin{array}{l}15 \\
22 \\
64\end{array}$ & $\begin{array}{l}29 \\
27 \\
44\end{array}$ \\
\hline $\begin{array}{l}\text { Sollicitation pour montrer aux collègues comment faire } \\
\text { Souvent } \\
\text { Parfois } \\
\text { Jamais }\end{array}$ & $\begin{array}{l}32 \\
49 \\
19\end{array}$ & $\begin{array}{l}35 \\
49 \\
16\end{array}$ & $\begin{array}{l}28 \\
47 \\
25\end{array}$ & $\begin{array}{l}34 \\
48 \\
17\end{array}$ \\
\hline $\begin{array}{l}\text { Sollicitation pour résoudre des problèmes, des conflits... } \\
\text { Souvent } \\
\text { Parfois } \\
\text { Jamais } \\
\text { Influence sur augmentation et promotion des subalternes (oui) }\end{array}$ & $\begin{array}{l}32 \\
36 \\
32 \\
57\end{array}$ & $\begin{array}{l}32 \\
41 \\
27 \\
55\end{array}$ & $\begin{array}{l}18 \\
42 \\
40 \\
32\end{array}$ & $\begin{array}{l}31 \\
41 \\
28 \\
54\end{array}$ \\
\hline Evaluation du travail des subalternes (oui) & 13 & 15 & 4 & 15 \\
\hline
\end{tabular}

Sources : d'après l'enquête COI 2006, nos traitements. 
Cet écart important entre d'un côté les représentations des modèles de la segmentation des marchés et de l'entreprise flexible d'une part, et les pratiques des entreprises de l'autre peut être interprété de manières différentes.

Ainsi, l'étude détaillée de certains salariés MAD, notamment les informaticiens employés par des Société de Service d'Ingénierie en Informatique (SSII) pour être mis à disposition d'entreprises multiples, a montré qu'ils constituent une maind'œuvre singulière, extérieure mais hautement qualifiée et fortement recherchée, dont le nomadisme d'une entreprise à l'autre peut être source d'intérêt non seulement pour les entreprises utilisatrices, mais aussi pour les travailleurs et les sociétés qui les emploient. Les informaticiens enrichissent leurs compétences en multipliant les missions dans des contextes variés ${ }^{7}$. Les SSII qui les emploient facturent chèrement leur expertise et leur adaptabilité, et les entreprises clientes tirent partie de leurs expériences, sans avoir à les licencier, en cas de baisse d'activité ou d'incompétence. Les informaticiens concernés correspondent ainsi au segment des marchés professionnels, le marché dit des compétences (Fondeur et Sauvat, 2002). Bien que leur niveau de qualification soit élevé et recherché, ils peuvent être ainsi externalisés et déplacés d'une entreprise à une autre, car leurs savoirs et leurs techniques sont relativement transférables ${ }^{8}$.

L'externalisation in situ d'expertise informatique sur des durées plus ou moins provisoires peut donc fonctionner pour des informaticiens en régie. Mais il est permis de douter de la pertinence de cette pratique pour d'autres cadres dont les responsabilités managériales sont beaucoup plus étendues. La situation est ainsi nettement plus étrange pour les salariés MAD en situation de management : les chefs d'équipe, notamment, qui représentent $30 \%$ de la population des MAD étudiée ici.

L'attribution de responsabilités managériales (encadrement d'équipe, explication du travail à réaliser aux subalternes, répartition du travail, résolution de problèmes ou de conflit, évaluation du travail réalisé par les subalternes, etc.) aux salariés en MAD est le point le plus surprenant de ce travail de découverte de l'identité et de l'activité de ces salariés atypiques. Un travail de management ou d'encadrement constitue à l'évidence un travail à fort enjeu pour l'entreprise qui requiert de nombreuses compétences spécifiques et stratégiques (Mispelblom Beyer, 2008) et qui justifient des écarts de rémunération sensibles par rapport à des exécutants

\footnotetext{
${ }^{7}$ Les caractéristiques démographiques de cette population d'informaticiens, typique des SSII, les prédisposent à bouger facilement. Cette main-d'œuvre est en effet jeune, sans enfants, citadine, masculine et très diplômée $(b a c+5)$. Leur propension à changer fréquemment d'environnement est d'autant plus grande, qu'ils prennent généralement eux-mêmes l'initiative de changer d'employeur (Fondeur et Sauvat, 2002).

${ }^{8} \mathrm{La}$ question de la transférabilité de leurs compétences mériterait un débat plus approfondi qui n'est pas dans le propos de cet article. Retenons l'idée que les langages informatiques, les systèmes d'information en général, ainsi que les contingences organisationnelles et sectorielles des entreprises dans lesquelles les TIC sont implantées peuvent limiter la capacité des informaticiens à intervenir dans n'importe quel contexte. Donc la transférabilité des experts informaticiens pour intervenir dans des entreprises différentes rencontre des limites certaines.
} 
(Létondal, 1997 ; Trouvé, 1996 ; Jacquemain, 1987). Par ailleurs, la fonction de management comporte intrinsèquement une dimension collective. Manager suppose de connaître son équipe. Cette connaissance s'inscrit dans le temps et nécessite fondamentalement de la coopération, de la proximité, certains ajoutent de la solidarité (le sentiment d'être unis par un objectif et un sort commun), voire de la confiance. Enfin, les encadrants, même s'ils ne sont «que » chef d'équipe disposent d'une délégation de pouvoir des dirigeants, dont ils sont de fait les représentants. La situation apparaît d'autant plus surprenante que ces salariés MAD exercent des responsabilités d'encadrement au nom d'un dirigeant d'entreprise, le client, dont ils ne sont pas les employés.

Dans son analyse consacrée aux cadres et aux ressources intellectuelles, X. Baron (2003) insiste pour dire que ce type de ressources humaines est essentiel à la production de valeurs dans l'entreprise, qu'ils sont le relais de la stratégie, qu'ils ne sont pas substituables, qu'ils sont au cœur d'interactions complexes et à haute valeur ajoutée: «Experts, engagés dans de multiples interactions en système, nécessaires au traitement de l'information, les producteurs intellectuels sont les seuls à pouvoir dire (et à faire ensuite) ce qu'il faut pour atteindre les résultats attendus. Le levier de l'animation hiérarchique se déplace ainsi de la définition des modes opératoires à une meilleure définition/déclinaison des objectifs ${ }^{9}$ : en facilitant la compréhension et en développant la capacité d'interprétation des objectifs généraux de l'entreprise par un effort de traduction et de réappropriation, à tous les niveaux. Il ne s'agit pas simplement d'améliorer la communication pour une meilleure motivation. Il s'agit d'accroître la capacité stratégique des salariés pour leur permettre de concevoir, de s'auto-organiser, d'arbitrer entre de multiples priorités et finalement d'être plus pertinents, à compétence et engagement équivalent » (Baron, 2003, p. 113). Les enjeux dont sont porteurs les cadres ou les ressources intellectuels sont manifestement énormes. Les théories de la segmentation ont complètement raison de les placer parmi les insiders, dans le marché primaire. Or, et c'est tout le paradoxe, une partie non négligeable des salariés MAD (les $20 \%$ de cadres, les $30 \%$ de chefs d'équipe) exercent des responsabilités importantes au sein d'entreprise(s) sans y être intégrés sur le modèle des insiders.

En privilégiant une recherche de flexibilité, de réduction des coûts (qui reste à démontrer selon la nature des coûts en question : immédiats ou différés, directs ou indirects, évidents ou cachés), de réduction de toute forme possible d'irréversibilité, de contrainte, ou de rigidité, on mesure à quel point, à la lecture des enjeux énoncés ci-dessus, les entreprises qui choisissent d'externaliser des fonctions d'expertise, d'encadrement ou de management prennent des risques que nous allons analyser maintenant.

\footnotetext{
9 Dans une note en bas de page importante (page 113), l'auteur précise que c'est à un niveau quasiment individuel qu'il est question de fournir les repères nécessaires à un travail quotidien pertinent. Des interactions individuelles et quotidiennes confirment bien la nécessité d'un rapport de proximité durable entre managers et managés.
} 


\section{Les difficultés soulevées par la mise à disposition de salariés qualifiés}

L'enquête COI fournit d'autres renseignements importants concernant le ressenti des salariés MAD en matière d'intégration dans les collectifs de travail, de sécurité professionnelle (risque de perdre son emploi dans l'année qui vient), et d'implication au travail.

Aux données statistiques de l'enquête COI, nous allons mêler des données plus qualitatives, en l'occurrence des verbatim de salariés MAD. Les témoignages en question proviennent tantôt d'études de terrains menées par des chercheurs en sciences sociales, tantôt d'enquêtes à caractère journalistique.

\subsection{Une identité organisationnelle floue associée à un relatif isolement dans le travail}

La situation des salariés qualifiés mis à disposition apparaît d'autant plus surprenante et lourde de conséquences pour les entreprises, qu'ils occupent une position ambiguë entre deux organisations. Qui est leur réel chef, c'est-à-dire qui les encadre et contrôle leur travail ? Quels sont leurs collègues réels, qui leur apporte une aide en cas de besoin ? Les réponses émises par les salariés MAD sont différentes de celles des permanents et suggèrent qu'ils ne savent plus toujours très bien à quel collectif de travail ils appartiennent. C'est la question de l'affiliation sociale (sentiment d'appartenir à un groupe et d'avoir des contacts sociaux enrichissants) nécessaire à la motivation au travail et donc à la performance (Gagné et Déci, 2005), qui est ici perturbée dans le cas des MAD. Un verbatim extrait d'une étude portant sur les normes d'emploi et les formes de travail dans les SSII est à ce titre particulièrement révélateur :

«Le problème quand tu es employé en régie, c'est que tu ne vois personne, tu ne sais plus pour quelle boite tu travailles; c'est pour le client, non ce n'est pas pour le client, tu n'es pas le salarié du client mais de la SSII, mais la SSII, tu ne la vois pas [...]. Tu ne sais plus quelle boite du défends. Tu défends le client ou la SSII? Tu ne sais pas, tu n'appartiens à personne, tu n'as plus de structure... » témoignage de salarié de start-up ayant eu plusieurs expériences en SSII, relaté dans Fondeur et Sauvat (2002, p. 45).

Ce témoignage fait écho à une mise en garde des juristes concernant la mise à disposition : «Il n'existe pas de situation plus grave pour le salarié que lorsqu'on ne peut désigner son réel employeur. L'effacement apparent de certains caractères distinctifs du statut salarial (la subordination), loin de comporter les prémices d'une quelconque «libération» du travailleur ou de l'allègement du pouvoir patronal, conduit seulement au déplacement de ce pouvoir hors du cadre juridique où il est supposé s'exercer »(Lyon-Caen et De Maillard, 1981, p. 326).

De manière générale, comparés aux autres salariés, les travailleurs mis à disposition considèrent être moins intégrés dans les collectifs de travail : ils disent travailler moins régulièrement avec d'autres collègues ou un supérieur 
hiérarchique, que les salariés internes. Les MAD sont ainsi plus nombreux à devoir travailler seuls que les permanents en CDI et à temps plein $(9 \%$ contre 3 $\%)$. Par ailleurs, les MAD sont plus nombreux à être exclus des temps de réunion de travail : $39 \%$ des MAD sont en effet dans ce cas, contre $25 \%$ des permanents à temps complet.

Une explication possible à ce relatif isolement des salariés mis à disposition réside dans l'incertitude concernant la durée de la coopération, ainsi que le suggère le témoignage suivant : "Le fait d'être sous-traitant me protège : ils ne me demandent pas autant que si j'étais membre de C (l'entreprise cliente). Mais ça me dessert aussi : je ne fais pas les calculs prospectifs avec eux car ils ne sont pas sûrs que je serai encore là dans deux ans ».

Ce flou identitaire peut s'accompagner d'une ostensible mise à distance des salariés mis à disposition de la part des salariés titulaires eux-mêmes. Nous en voulons pour preuve le témoignage suivant :

"Pendant neuf ans, j'ai été détaché (en tant qu'ingénieur informatique) dans la même entreprise, avec des contrats renouvelés tous les ans. Les salariés nous appelaient les "sous-traitants", une manière de nous tenir à distance.(...) Dans le milieu de la sous-traitance [sur-site], il faut savoir être agressif, se battre, se valoriser en permanence. C'est nécessaire pour que le client renouvelle le contrat avec votre employeur. Et à plus forte raison, quand on veut se faire embaucher [par l'entreprise cliente](...) Être sous-traitant, c'est être constamment en position de demandeur d'emploi à l'intérieur de l'entreprise. J'étais constamment en position de faiblesse, au service des gens, sans que la réciproque fonctionne. Obligé d'être pro, sans jamais se plaindre" ${ }^{10}$.

Cela est d'autant plus paradoxal que les salariés MAD occupent pour une proportion non négligeable d'entre eux des postes à responsabilité dans les entreprises clientes, comme dans l'exemple de cet ingénieur informaticien: comment exercer des responsabilités, collaborer, parfois même encadrer des salariés des entreprises clientes, alors qu'il peut exister une mise à distance entre le personnel interne (insiders) et ces prestataires externes (outsiders) ?

Ces exemples ne sont pas forcément représentatifs de toutes les situations de mise à disposition. Mais ils permettent de montrer certaines des problématiques induites par la mise à disposition de personnel, surtout quand celle-ci concerne des personnes qualifiées exerçant des responsabilités importantes. La question du soutien social, qui renvoie à l'aide et au support dont peut bénéficier le salarié de la part de ses collègues ou de sa hiérarchie, a été explicitement intégrée comme variable pouvant affecter le stress professionnel, les risques psychosociaux et donc porter atteinte à la santé au travail (Guignon et al. 2008). Ce stress est particulièrement important lorsque le sentiment de responsabilité est élevé (inquiétude des conséquences d'une erreur dans le travail) et que le soutien social est faible. Or, ces deux conditions sont réunies chez les MAD puisqu'ils se

10 Témoignage paru dans Libération, le 27 avril 1998, dans un dossier Emploi intitulé : «Contrats : l'entreprise s'éclate». 
sentent à la fois responsables (cf. chiffres du tableau 2), mais plus isolés, moins soutenus par les collègues et leur hiérarchie, et qu'ils sont parfois tenus à distance par les salariés internes (titulaires). Or, le stress dégrade la satisfaction au travail, ce qui peut expliquer une implication moins importante des MAD par rapport aux salariés titulaires à temps complet (nous y revenons ci-après infra $\$ 3.3$ ).

Pour des salariés mis à disposition chez un seul et même client depuis plusieurs années, comme dans l'exemple de l'ingénieur informaticien rapporté ci-dessus, qui exerce vraiment la prérogative d'employeur ? Qui dirige de fait les tâches réalisées par le salarié du prestataire ? Cette fonction de contrôle des salariés MAD davantage exercée par des personnes extérieures à l'entreprise (employeuse) confirme la dislocation du lien organisationnel. Faut-il s'étonner que les salariés MAD finissent pas s'identifier davantage à l'entreprise cliente qu'à l'entreprise employeuse ?

Un témoignage rapporté dans le cadre d'une commission de concertation auprès d'une entreprise sous-traitante qui met à disposition des salariés chez une entreprise cliente depuis de nombreuses années, confirme l'ambiguïté de la situation. A noter, en particulier, la distinction nette opérée dans les propos du témoin, entre le patron employeur (distant) et le chef-manager de proximité de qui le témoin se sent proche, mais sans faire partie officiellement de la même entreprise :

"J'ai été recruté par C (l'entreprise cliente) qui a demandé à l'entreprise $S$ (l'entreprise sous-traitante) de m'embaucher " (...) Je vois mon patron (employeur officiel) une fois par an, c'est moi qui demande à avoir mon entretien annuel d'évaluation »(...) Pour mener cet entretien d'évaluation, mon patron contacte mon chef (le manager de proximité de l'entreprise cliente) et lui demande comment j'ai travaillé. (...) "Si mon entreprise n'est pas retenue dans le prochain appel d'offre, je pense que je resterai quand même chez $C$ (entreprise cliente), car mon chef dira à la nouvelle entreprise qu'il a besoin de moi et elle m'embauchera " (...) Parfois je bosse avec un gars et j'apprends quelque temps plus tard qu'on est de la même boîte... ».

\subsection{Une intégration professionnelle moins assurée pour les MAD}

Les MAD ressentent aussi davantage d'incertitudes quant à leur activité future. Ils expriment quasiment la même probabilité de perdre leur emploi l'année suivante que les salariés internes en CDD, à temps partiel ou en contrat d'apprentissage (cf. tableau 3 ci-dessous). 
Tableau 3 - Répartition en pourcentage des salariés des entreprises de plus de 20 personnes, selon leur type d'emploi et leur sentiment de fragilité professionnelle (risque perçu de perdre son emploi dans l'année qui vient)

\begin{tabular}{|l|c|c|c|}
\hline & MAD & $\begin{array}{c}\text { Salariés internes en } \\
\text { CDI à temps complet }\end{array}$ & $\begin{array}{c}\text { Autres salariés } \\
\text { internes }\end{array}$ \\
\hline Précarité /risque de perte de son emploi dans & & & \\
I'année qui vient & 16 & & 17 \\
$\quad$ Oui ou peut-être & 74 & 81 & 75 \\
Non ou probablement pas & 10 & 10 & 8 \\
Ne sait pas & & \\
\hline
\end{tabular}

Sources : d'après l'enquête COI 2006, nos traitements.

C'est sans doute autour de cette question qu'apparaît le plus l'écartèlement des MAD entre deux statuts antagonistes: d'un côté, les salariés détenteurs de compétences précieuses pour l'entreprise qui devrait, en principe, les embaucher pour les placer à l'intérieur du noyau dur, parmi les insiders (ce qui n'est pourtant pas le cas, puisque ces MAD ne font pas partie des effectifs de l'entreprise cliente). Et de l'autre, les ressources humaines clairement périphériques dont l'entreprise a besoin de temps en temps, et dont les compétences sont banalisées : typiquement les intérimaires (voir la figure 1 inspirée du modèle de l'entreprise flexible d'Atkinson).

En d'autres termes, un MAD (du moins, pour les $20 \%$ qui sont cadres ou les 30 $\%$ qui sont chefs d'équipe) remplit des fonctions stratégiques au sein de l'entreprise cliente. Mais il ressent le même risque de perdre son emploi que les ressources explicitement précaires que sont les intérimaires ou les CDD qui eux sont nettement moins qualifiés que les MAD.

L'explication de ce sentiment de précarité provient du fait que la relation d'emploi pourtant stable avec l'entreprise sous-traitante $(78 \%$ des salariés mis à disposent d'un CDI) est conditionnée par un lien commercial intrinsèquement fragile avec l'entreprise donneuse d'ordres. Certes, le salarié dont la mise à disposition s'achève revient dans l'entreprise qui l'emploie, et celle-ci continue à lui verser un salaire entre les missions, en le formant ou lui cherchant d'autres mises à disposition ; mais ces périodes d'inter-missions sans mise à disposition, et donc sans facturation, ne peuvent pas durer éternellement. D'où la crainte permanente d'une interruption des mises à disposition dépendantes d'une relation commerciale qui peut être remise en cause à tout moment.

L'exercice de responsabilités managériales importantes est-il compatible avec le risque perçu de perdre son emploi à court terme ? Il est permis d'en douter.

\subsection{Une implication dans le travail mitigée pour les MAD}

L'une des dernières questions (question 92) posée dans l'enquête COI porte sur une question centrale de la GRH. Cette question est la suivante : «Dans votre travail, vous impliquez-vous: peu, juste ce qu'il faut, beaucoup?» La formulation de la question est certes un peu simpliste, mais elle permet d'agréger divers éléments permettant de mesurer et de comparer le degré d'implication dans le travail en fonction du statut d'emploi. 
Tableau 4 - Répartition en pourcentage des salariés des entreprises de plus de $\mathbf{2 0}$ personnes, selon leur type d'emploi et leur implication dans le travail

\begin{tabular}{|l|c|c|c|}
\hline & MAD & $\begin{array}{c}\text { Salariés internes en } \\
\text { CDI à temps complet }\end{array}$ & $\begin{array}{c}\text { Autres salariés } \\
\text { internes }\end{array}$ \\
\hline Implication dans son travail & 2 & & 2 \\
Peu & 31 & 26 & 42 \\
Juste ce qu'il faut & 68 & 73 & 55 \\
Beaucoup & & \\
\hline
\end{tabular}

Sources : d'après l'enquête COI 2006, nos traitements.

Les chiffres du tableau 4 montrent un degré d'implication intermédiaire, c'est-àdire que les salariés MAD sont plus impliqués dans leur travail que les salariés internes à temps partiel, en CDD ou en contrat d'alternance, mais moins que les salariés internes à temps complet. Ce score intermédiaire est conforme à leur positionnement professionnel ambigu: pour les salariés mis à disposition, l'exercice du travail (chez le client) est déconnecté de leur relation d'emploi (avec leur employeur).

En effet, les salariés MAD sont relativement précaires car soumis à l'incertitude d'une relation commerciale entre leur employeur et l'entreprise cliente où ils sont mis à disposition: "Une direction d'entreprise maîtrise souvent mieux un fournisseur qu'un salarié car elle dispose de moyens de pression plus efficaces. Par ailleurs, en cas de baisse d'activité, il est plus facile de se séparer d'un soustraitant que de licencier une équipe $»$. (Baron, 2003, p. 117). A ce titre, tout comme les salariés contingents (cf. CDD, intérimaires), il se peut que les MAD ressentent un défaut de reconnaissance ou d'estime; ils peuvent se sentir découragés par le traitement impersonnel dont ils font l'objet au travail (cf. l'ingénieur informaticien en régie qui déplore que les salariés titulaires marquent une distance avec lui, en l'appelant « le sous-traitant») ; se sentir menacés dans leur emploi d'autant plus qu'ils cherchent, pour certains, à se faire embaucher par l'entreprise cliente.

La fin de leur contrat et l'anticipation de leur non-renouvellement conduit peutêtre les MAD à moins s'engager, comme le suggère C. Manville, qui a observé que des relations «dont la durée est a priori limitée dans le temps pouvaient inciter les salariés à ne pas s'inscrire durablement dans l'action collective ou à diminuer leurs efforts, à moins s'impliquer, etc. ( (Manville 2005, p. 62-64). C. Manville mobilise le concept de justice organisationnelle pour analyser les effets pervers d'une flexibilité qui s'établit aux dépens des personnes. Ce concept de justice organisationnelle, qui ferait défaut s'agissant des MAD, semble approprié pour comprendre une implication dans le travail que nous qualifions de « retenue » s'agissant des MAD.

En même temps, de par leur formation (études supérieures, statut cadres, exercice de responsabilité, etc. pour une partie non négligeable d'entre eux), les MAD peuvent manifester une éthique d'implication et d'engagement conforme aux personnes qualifiées exerçant des responsabilités significatives. 
D'où le tiraillement possible entre différentes identités professionnelles (Sainsaulieu, 1977) antagonistes: celles d'un cadre, d'un expert, ou d'un manager d'un côté ; et de l'autre, celle d'un salarié pas complètement intégré dans l'entreprise où il travaille, en sentiment de précarité, ni dans les collectifs de travail (identité fusionnelle dans les groupes restreints). Tiraillement qui peut expliquer ce score intermédiaire d'implication dans le travail concernant les salariés MAD.

\subsection{Les symptômes d'un malaise professionnel touchant les MAD}

Même si la question n'a pas été abordée explicitement dans l'enquête COI, les difficultés que nous venons de présenter (identité organisationnelle floue associée à un relatif isolement dans le travail ; risque perçu de perdre son emploi ; implication mitigée dans le travail) suggèrent la possibilité d'un malaise dans le travail dont pourraient souffrir les salariés MAD. Lequel est forcément problématique au regard de la performance attendue des collaborateurs en général, mais aussi de ces salariés MAD surtout ceux qui exercent des responsabilités importantes (cadres et chefs d'équipe).

Ce sont principalement les propos exprimés dans les verbatim des salariés MAD qui nous mettent sur la voie d'un possible malaise professionnel dont souffriraient les MAD. Lequel contribuerait à expliquer l'implication inférieure de ces derniers par rapport aux salariés titulaires à temps complet.

Un malaise professionnel peut être défini comme le résultat d'un conflit entre les valeurs professionnelles (engendrées notamment par les rôles assumés et les formations reçues) et les exigences organisationnelles. Huit points de tension sont particulièrement pris en considération. Il s'agit de la nature du travail, l'autonomie, la participation, le style de gestion du supérieur, les conditions de travail, le déroulement de carrière, la reconnaissance et l'éthique (Guérin et al., 1996).

Il est intéressant de noter que la recherche sur le malaise professionnel que nous mobilisons ici a porté sur la population des cadres. Les auteurs insistent notamment sur l'importance de la loyauté, de l'implication à l'organisation comme des éléments structurant favorablement l'identité des cadres et éloignant donc ces derniers d'un risque de malaise professionnel : «En premier lieu, le cadre est au service de l'organisation. Investi d'une autorité formelle par les propriétaires, il doit assurer la survie de l'organisation en produisant d'une manière efficace des biens et des services spécifiques (...). Dans un tel contexte, il n'est pas étonnant que la culture managériale soit marquée du sceau de la loyauté et de l'identification à l'organisation (...) » (ibid., p. 5).

Quand on rapporte ce raisonnement aux propos exprimés par l'ingénieur informaticien en régie que nous avons déjà cité: "Le problème quand tu es employé en régie, c'est que tu ne vois personne, tu ne sais plus pour quelle boite tu travailles; c'est pour le client, non ce n'est pas pour le client, tu n'es pas le salarié du client mais de la SSII, mais la SSSI tu ne la vois pas [...]. Tu ne sais plus quelle boite du défends. Tu défends le client ou la SSII ? Tu ne sais pas, tu 
n'appartiens à personne, tu n'as plus de structure... » témoignage de salarié de start-up ayant eu plusieurs expériences en SSII, rapporté par Fondeur et Sauvat (2002, p. 45) ; la culture managériale nécessaire à l'identité des cadres fait ici clairement défaut pour les salariés MAD. Et cette difficulté à s'identifier à une structure précise peut générer une forme de malaise professionnel.

De la même façon, quand un autre ingénieur en MAD se fait traiter de soustraitant par les salariés titulaires ( Les salariés nous appelaient les sous-traitants, une manière de nous tenir à distance (...) J'étais constamment en position de faiblesse, au service des gens, sans que la réciproque fonctionne» (cf. témoignage cité plus haut); cela montre à nouveau une forme de discrimination à l'endroit des $\mathrm{MAD}$, une manière explicite de ne pas les intégrer à la communauté dans laquelle ils travaillent pourtant de façon pérenne, mais sans en faire vraiment partie (la mise à disposition dans la même entreprise, à temps plein, a pourtant duré neuf ans en l'occurrence). La mise à distance, une certaine forme d'ostracisme, le défaut d'intégration au collectif, sont générateurs de malaise professionnel. Lequel peut expliquer un degré d'implication dans le travail moins important chez les MAD que parmi les salariés intégrés à l'entreprise à temps complet.

Une autre source possible de tension et donc de malaise professionnel résulte de la tendance au traitement uniforme et anonyme. Etre appelé « sous-traitant» au lieu du nom ou prénom de la personne est typique de ce traitement anonyme dont se plaint l'ingénieur en régie. «En conformité avec le modèle des relations humaines, de tels comportements laissent croire au professionnel qu'il n'est pas apprécié, qu'il est considéré comme immature ou qu'il n'est qu'un rouage négligeable dans l'atteinte des objectifs organisationnels. Les relations déshumanisantes qu'entretiennent certains cadres à l'endroit de leurs subordonnés laisseraient ces derniers insatisfaits et peu portés à s'impliquer davantage. Ainsi le manque de consultation est fréquemment dénoncé par les professionnels. Le manque de confiance que leur témoignent les cadres est un autre point qui déçoit certains professionnels » (ibid. p. 10). Cette analyse fait écho au travail davantage solitaire des MAD et à la moindre participation de ces derniers à des réunions de travail.

Une source supplémentaire de malaise professionnel génératrice d'insatisfaction et de désimplication renvoie à la problématique de la fragilité professionnelle, question que nous avons abordée plus haut : $16 \%$ des MAD expriment le risque de perdre leur emploi dans l'année qui vient (contre $9 \%$ pour les salariés internes en CDI à temps complet et $17 \%$ pour les autres salariés internes). «La précarisation croissante des milieux professionnels est également dénoncée. Il serait impossible de se concentrer sur sa pratique professionnelle, voire de développer un minimum d'identification organisationnelle, lorsqu'on n'est pas assuré d'une sécurité d'emploi » (ibid.p. 10).

Cette hypothèse d'un malaise professionnel touchant les MAD que nous venons d'argumenter compromet gravement les vertus de cette flexibilité externe (Everaere, 2012) et engendre des inconvénients sérieux dont devraient tenir compte les tenants d'une externalisation à outrance de l'emploi, surtout si les 
postes en question confiés aux MAD nécessitent des compétences et des responsabilités importantes pour l'entreprise utilisatrice.

\section{Conclusion}

Jusqu'où la recherche de flexibilité poussera-t-elle les entreprises ? Après l'entreprise sans frontière, l'entreprise sans usine («the fabless company », selon Alcatel), demain l'entreprise sans salarié ? Nous touchons avec ce profil de salarié MAD qualifié et externalisé, durablement implanté dans les locaux de l'entreprise cliente, aux limites de ce que nous enseignent les théories disponibles concernant les représentations de l'entreprise flexible.

Certes, au vu des chiffres fournis par l'enquête COI 2006, ces salariés MAD sont dans leur globalité relativement peu nombreux : 769 unités de l'échantillon, soit $5,4 \%$ de la population enquêtée. «Seuls » $20 \%$ d'entre eux sont cadres et $30 \%$ exercent des responsabilités hiérarchiques. Ils ne sont ainsi qu'entre 1 et $2 \%$ à invalider les modèles théoriques disponibles. Compte tenu de leur profil, qualifié pour une partie significative d'entre eux et de la durabilité des besoins, l'entreprise devrait les embaucher pour en faire des salariés titulaires, des insiders. Pourtant, elles ne le font pas.

Résoudre ce paradoxe nécessiterait d'interroger les responsables des entreprises qui font le choix d'externaliser et de confier aux salariés d'entreprises extérieures des missions importantes (s'agissant des $20 \%$ de salariés MAD cadres et des 30 $\%$ de managers) et durables chez elles. Question essentielle que nous ne permet pas de traiter l'enquête COI 2006 qui dans ce domaine ne porte que sur le point de vue exprimé par les salariés ${ }^{11}$.

La situation organisationnelle et professionnelle des salariés MAD nous parait cependant intéressante pour instruire la question de la portée et des limites de cette forme particulière de flexibilité externe quasi-internalisée. L'implication mitigée des MAD, implication pourtant essentielle à la performance des entreprises, constitue l'un des points de vigilance importante dont devraient tenir compte les entreprises tentées par cette forme d'externalisation hybride.

\section{Références}

Altkinson, J. (1984), «Manpower Strategies for Flexible Organisations », Personal Management, $\mathrm{n}^{\circ} 16-8$, août, p. 28-3.

Baron X. (2003), «De la gestion des cadres à la gestion des ressources intellectuelles », in Allouche J. (coord), Encyclopédie des ressources humaines, Vuibert.

\footnotetext{
${ }^{11}$ L'enquête COI 2006 comportait deux volets : un premier sur les salariés et un second sur les entreprises qui les emploient. Cependant la plupart des questions qui nous ont intéressés ici n'ont pas été abordées avec les entreprises employeur.
} 
Barreyre, P.-Y. (1992), «La sous-traitance à l'heure des nouvelles politiques d'impartition », in J.P. Helfer et J. Orsoni (Éds.), Encyclopédie du management, volume 2, Vuibert.

Cadin, L. (1997), «Faut-il sortir la GRH de ses frontières ? », in P. Besson (coord.), Dedans, dehors, les nouvelles frontières de l'organisation, Vuibert.

Capet, M. (1989), "Sous-traitance : aspects économiques et financiers », in P. Joffre et Y. Simon (Éds.), Encyclopédie de gestion, volume 3, Vuibert.

Doeringer, P. et M. Piore (1971), Internal labor market and manpower analysis, New York : Sharp.

Domens, J. (2010), «L'emploi intérimaire au premier trimestre 2010 : l'intérim poursuit sa remontée $»$, Dares Indicateurs, juin, $\mathrm{n}^{\circ} 40$.

Everaere, Ch. (2012), «La flexibilité appliquée aux ressources humaines: compatibilités et contradictions », Revue Française de Gestion, n 221, février.

Everaere, Ch., Grimand A. et Laviolette E.M., (2003), «Formation, conditions de travail et relations sociales dans les sociétés de services informatiques », Education Permanente, $\mathrm{n}^{\circ}$ 155/2003-2.

Finot J. (2012), «L'intérim en 2011 : croissance soutenue », DARES Analyses, juin, $\mathrm{n}^{\circ} 042$.

Fondeur, Y. et C. Sauvat, (2002), « Normes d'emploi et marché du travail dans les métiers liés aux technologies de l'information », Document d'études DARES, $\mathrm{n}^{\circ} 63$, novembre.

Gagné, M., \& Deci, E. L. (2005). "Self-determination theory and work motivation". Journal of Organizational Behavior, 26, 331-362.

Gazier, B. (1993), Les stratégies des ressources humaines, La Découverte, coll. Repères.

Guérin G., Wils T., Lemire L. (1996), «Le malaise professionnel : nature et mesure du concept », Relations industrielles / Industrial Relations, vol. 51, $\mathrm{n}^{\circ} 1$, p. 62-96.

Guignon N., Niedhammer I., Sandret N., (2008), «Les facteurs psychosociaux au travail. Une évaluation par le questionnaire de Karasek dans l'enquête SUMER 2003 », Premières Informations et Premières Synthèses, DARES, mai 2008, n $22-1$.

Jacquemin, R. (1987), «La fonction de maitrise et son évolution », in C. LevyLeboyer et J-C. Sperandio (Eds.). Traité de psychologie du travail, Paris : PUF.

Letondal, A-M. (1996). L'encadrement de proximité. Quels rôles dans les changements d'organisation, ANACT, coll. Outils et Méthodes.

Lyon-Caen, A. et J. de Maillard (1981), «La mise à disposition de personnel », Droit Social, ${ }^{\circ} 4$, avril. 
Maillard, J. (de), P. Mandroyan, JP. Plattier et T. Priestley (1979), « L'éclatement de la collectivité de travail : observations sur les phénomènes d'extériorisation de l'emploi », Droit Social, n ${ }^{\circ}$ 9-10, septembre-octobre.

Manville, C. (2005), «Les enjeux de la flexibilité quantitative du travail : l'apport de la théorie de la justice organisationnelle », Cahiers du Recherche du GRECO, Montpellier, $\mathrm{n}^{\circ} 20$, juillet - septembre.

Mispelblom Beyer, F. (2008), «Statuts et orientations dans les pouvoirs d'encadrement », Cadres et Sociétés, ${ }^{\circ} 2$, novembre.

Sainsaulieu R., L'identité au travail, Presses de la FNSP, 1977, réed. 1985.

Trouvé, Ph. (1996), «La fin des contremaîtres traditionnels », Revue Française de Sociologie, ${ }^{\circ} 37-2$, pp. 287-308.

Williamson, O. (1975), Markets and Hierarchies. Analysis and antitrust Implications, The Free Press, New York. 